\title{
Evaluación de docentes en programas de posgrado
}

\section{Assessment of teachers in postgraduate programs}

\author{
Ana C. Olascoaga' ${ }^{1}$ Ray Ticse Aguirre'1, José Caballero López¹
}

\section{Resumen}

Los profesores son parte fundamental de una escuela de medicina. A nivel de la residencia médica, la evaluación de la docencia clínica es una estrategia de mejoramiento de la educación que permite identificar las deficiencias que existen para superarlas mediante capacitación, así como reconocer las buenas prácticas docentes. Los modelos de evaluación docente utilizados en distintas facultades de medicina comprenden: la evaluación por pares, la autoevaluación, la evaluación basada en la opinión de los estudiantes y el portafolio docente. Con el fin de mejorar la calidad de la enseñanza y la calidad de los programas formativos en la residencia médica es necesario contar instrumentos de evaluación válidos, confiables, factibles y que cuenten con la aceptación de los profesores y residentes. Esta evaluación debe ser devuelta al docente mediante un proceso de retroalimentación efectiva y conectarse a un plan de desarrollo docente, de capacitación y de reconocimiento, enmarcado dentro de la carrera profesional. El sistema de evaluación y desarrollo docente debe partir de la institución universitaria responsable de la formación del médico residente.

Palabras clave: enseñanza; residencia médica; evaluación; desarrollo docente.

\begin{abstract}
Teachers are a substantial part of a medical school. In medical residency, the evaluation of clinical teaching is a strategy for improving education that allows establishing the deficiencies to overcome them through training and also to recognise good teachers. Teaching evaluation models used in different medical schools include peer evaluation, self-evaluation, evaluation based on the opinion of students and the teaching portfolio. To improve the quality of teaching and the quality of training programs in medical residences, it is necessary to have a valid, reliable, feasible teacher evaluation system that has the acceptance of teachers and residents. This evaluation must be returned to the teacher through an effective feedback process and also linked to a faculty development program and a recognition plan framed within the professional career. The teaching assessmente and development system must come from the university responsible for the resident training.
\end{abstract}

Keywords: Teaching; residency; assessment; faculty development.

Fecha de envío: 2021-10-26 - Fecha de aceptación: 2021-12-03

\section{Introducción}

La educación médica de calidad se basa en los siguientes fundamentos: el diseño de un currículo centrado en el alumno que responda a las necesidades de la sociedad, la formación de un equipo docente competente y comprometido, el uso de tecnología educativa innovadora y la evaluación de la calidad de la educación.

Los profesores son parte fundamental de una escuela de medicina y son el motor del aprendizaje de los alumnos. A nivel de posgrado, específicamente en la residencia, los médicos docentes son quienes cumplen un papel decisivo en la enseñanza de los residentes en los lugares de trabajo: centros de atención primaria, clínicas y hospitales. La calidad de la atención en salud depende esencialmente de la calidad de la educación médica.

La evaluación es "un proceso sistemático que permite la integración de la información cualitativa y cuantitativa para determinar el valor o mérito de un objeto, donde a partir de la información recopilada se emiten juicios que orientan la toma de decisiones con fines de

(1) Unidad de Educación Médica, Facultad de Medicina. Universidad Peruana Cayetano Heredia. Lima. Perú.

Autor de correspondencia: ana.olascoaga.m@upch.pe 
mejora" (Flores et al., 2011). En la residencia médica, la evaluación de la docencia clínica es una estrategia de mejoramiento de la educación porque permite conocer las deficiencias para superarlas mediante capacitación y puede brindar un reconocimiento a las buenas prácticas docentes y socializarlas e impulsarlas. Los profesores pueden desarrollar su labor de manera más eficiente si son sometidos a un proceso de evaluación de su desempeño y se les brinda retroalimentación asertiva, oportuna y efectiva. Así, la implementación de una evaluación estandarizada de los docentes de una facultad de medicina puede constituirse en una parte esencial de la evaluación de la calidad de la enseñanza y por tanto en una herramienta para el mejoramiento continuo.
En el presente artículo se revisan los roles del docente de la residencia médica, las características de la evaluación en este nivel de la educación médica y los instrumentos utilizados, los sesgos, las barreras encontradas y finalmente el planteamiento de la evaluación docente como una cultura de los programas de residencia médica.

\section{Roles del docente de residencia médica}

Harden y Crosby describieron hace más de veinte años doce roles docentes, agrupados en seis áreas (Harden \& Crosby, 2000):

Tabla 1: Roles docentes. Adaptación del texto de AMEE Guide №20 (Harden \& Crosby, 2000).

\begin{tabular}{|c|c|c|}
\hline Relación docente / estudiante & Área & Rol docente \\
\hline \multirow{6}{*}{ Directa } & \multirow{2}{*}{ Proveedor de información } & Conferencista \\
\hline & & Profesor clínico \\
\hline & \multirow{2}{*}{ Modelo a seguir } & Modelo a seguir como docente \\
\hline & & Modelo a seguir como médico \\
\hline & \multirow{2}{*}{ Facilitador } & Mentor \\
\hline & & Facilitador del aprendizaje \\
\hline \multirow{6}{*}{ Indirecta } & \multirow{2}{*}{ Evaluador } & Evaluador del alumno \\
\hline & & Evaluador del currículo \\
\hline & \multirow{2}{*}{ Planificador } & Planificador del currículo \\
\hline & & Organizador de curso \\
\hline & \multirow{2}{*}{ Desarrollador de recursos } & Productor de guías de estudio \\
\hline & & Creador de material didáctico \\
\hline
\end{tabular}

Los docentes no cumplen todos los roles, sino que, según su posición, desempeñan una combinación de éstos. La mayoría de los docentes clínicos de residencia médica cumplen con los roles de profesor clínico, ser un modelo a seguir como médico y como docente, ser mentor, facilitador del aprendizaje y evaluador del alumno. Los jefes de programas de residencia médica ejercen los roles de planificador y evaluador de currículo. Los docentes deben reconocer sus roles asignados.

El docente de la residencia médica ejerce sus roles en diversas áreas reales de práctica asistencial: hospitalario, consulta externa o en trabajo comunitario; enseñando a los médicos residentes habilidades clínicas y de comunicación, razonamiento clínico y la ejecución de procedimientos, todo ello de acuerdo con el programa y el nivel formativo correspondiente. Pero también el docente es un modelo a seguir en su forma de actuar, debiendo realizar la práctica con profesionalismo, respetando los principios éticos y trabajando armónicamente en equipos interdisciplinarios.
La docencia debe estar centrada en los alumnos, teniendo como objetivos facilitar y fomentar el autoaprendizaje. El docente se convierte en un mentor en la medida en que mantiene un equilibrio entre independencia y supervisión, dispuesto a ser una guía, fuente de consejo y apoyo. Finalmente, cumple su rol de evaluador observando el desempeño de las habilidades del médico residente en la práctica clínica diaria.

El fin del acto médico es el bienestar del paciente. Durante la residencia médica se combina la docencia con el trabajo asistencial por lo que el docente debe dar prioridad a la atención del paciente sin menoscabar la docencia, así debe responder a ambas demandas y establecer un equilibrio entre ellas. Este trabajo exige experticia en medicina y destreza en docencia. En este entorno el docente debe establecer claramente las funciones de los residentes y sus objetivos de aprendizaje.

A diferencia de los alumnos de pregrado, quienes carecen de experiencia y conocimientos bien cimentados, los residentes son profesionales 
médicos que poseen un grado de conocimientos de base, habilidades ya adquiridas y un nivel de experiencia, por lo que el trato entre docente y residente debe ser más horizontal, prevaleciendo la valoración y el respeto por el otro. Paukert y Richards encuentran que existe una diferencia en la forma cómo los roles docentes son apreciados por los alumnos y los residentes, los alumnos de pregrado valoran más el rol de proveedor de información, mientras que los médicos residentes valoran más el rol de supervisor (Paukert \& Richards, 2000).

Las jornadas de trabajo durante la residencia, característicamente largas y complejas, requieren de la presencia continua del docente para garantizar la enseñanza y la supervisión, es función del docente aprovechar toda oportunidad para el aprendizaje.

Pero también hay algunas dificultades y limitaciones que debemos considerar. La residencia médica se desarrolla fuera de las aulas universitarias, en áreas clínicas, comunitarias u hospitalarias, por lo que el cumplimiento de los roles docentes es influenciado por el sistema de salud, el sistema educativo y la cultura organizacional local. Estas áreas clínicas, si bien cumplen funciones similares en la mayoría de países, difieren en sus características porque dependen de la organización del sistema de salud de cada país y de su nivel económico. Además, muchas veces los docentes de residentes son médicos asistenciales que coinciden con los residentes en el campo clínico y cumplen la función de tutor como una labor adicional no remunerada, sin tiempo protegido para ejecutarla y sin capacitación (Organización Panamericana de la Salud-OPS, 2011). También, en muchos ambientes clínicos existe una cultura jerárquica disfuncional, en algunas especialidades más que en otras, lo que genera un mal clima de aprendizaje tanto para los residentes como para los docentes (Salehi et al., 2020). Por todas estas consideraciones es válido afirmar que la evaluación docente sistemática juega un rol en reconocer las debilidades y limitaciones de algunos docentes y escenarios para abordar mejoras y por otro lado identificar fortalezas y desarrollos de otros docentes y escenarios que sirvan de modelo a seguir.

Así, la evaluación del desempeño docente es un proceso que debe ser llevado a cabo por las instituciones universitarias porque tienen la responsabilidad de proveer de oportunidades de capacitación en docencia a todos sus profesores involucrados en la formación de los médicos residentes. La información obtenida a través de la evaluación permite estratificar o personalizar las capacitaciones.

\section{Instrumentos de evaluación docente en la residencia médica}

Los modelos de evaluación docente utilizados en distintas facultades de medicina comprenden: la evaluación por pares, la autoevaluación, la evaluación basada en la opinión de los estudiantes y el portafolio docente.
- Evaluación por pares: Esta es una fuente valiosa de retroalimentación para el docente; promueve un diálogo entre el evaluador y el evaluado con una resultante mejora de la calidad de la enseñanza y el aprendizaje. Se puede realizar en escenarios clínicos a nivel ambulatorio u hospitalario; durante la visita médica, la consulta externa, discusiones clínicas, conferencias y otras actividades. Existen diversas rúbricas de evaluación creadas para cada situación docente (Newman et al., 2012). El docente evaluador debe prepararse para realizar la observación, evaluar y brindar el feedback (retroalimentación) a su colega de manera clara y positiva; así mismo, el docente evaluado debe prepararse para recibir el feedback con apertura y humildad. Un proceso colaborativo de observación entre pares en el escenario clínico de la residencia médica logra un impacto positivo en el crecimiento y desarrollo profesional de los educadores (Finn et al., 2011).

- Autoevaluación: Esta evaluación cumple una función formativa. Utiliza instrumentos que describen las conductas esperadas en el profesor, definiendo así un objetivo a cumplir. Al realizar la autoevaluación el docente tiene la oportunidad de reflexionar en su propio desempeño y de identificar sus debilidades, por lo tanto, lo apoya a realizar esfuerzos de aprendizaje autodirigidos. La mayoría de estudios publicados al respecto corresponden a validación de instrumentos y a la comparación entre los resultados de la autoevaluación y la evaluación por los residentes (Stalmeijer et al., 2010; Vaižgèlienè et al., 2017; Arah et al., 2011).

- Evaluación basada en la opinión de médicos residentes: La evaluación del docente es realizada por el residente, generalmente al final de un periodo de entrenamiento. Este tipo de evaluación es la más usada actualmente para la evaluación de los docentes en la residencia médica. La mayoría de los instrumentos se basa en una lista de ítems que son calificados mediante una escala de Likert. Los aspectos evaluados en los instrumentos se refieren predominantemente al uso de estrategias de enseñanza en servicio, el rol de facilitador del aprendizaje, el rol de modelo a seguir y la retroalimentación. Los resultados son utilizados para proporcionar retroalimentación formativa a los docentes, asignación de recursos, revisión anual del desempeño y promoción docente.

- El portafolio del docente médico: Es una colección de documentos que evidencian los esfuerzos, progresos y logros del médico en el área de enseñanza clínica. El docente puede incluir un registro de su actividad, sus capacitaciones, sus logros, innovaciones, premios y reconocimientos, así como reflexiones personales sobre sus motivaciones y experiencias. 
- Este instrumento permite recogery presentar evidencias sobre la efectividad de su enseñanza, reflexionar sobre las áreas de enseñanza que necesita optimizar, mantener un registro de la evolución de la enseñanza, compartir conocimientos y experiencia con pares y ser evaluado cualitativa y cuantitativamente por sus superiores (Matus et al., 2009). En muchas instituciones universitarias el portafolio es utilizado como parte de la evaluación anual del docente y para realizar los procesos de ratificación y promoción (Simpson et al., 2004).

Se debe garantizar que los instrumentos utilizados en la calificación sean válidos y confiables, además deben ser fáciles de aplicar, aceptables y de bajo costo. En el caso de evaluación realizada por estudiantes, se muestra diferentes niveles de validez y confiabilidad, y diversa extensión (Fluit et al., 2010). Podemos citar algunos instrumentos creados para posgrado: el Standford Faculty Development Program desarrollado en Estados Unidos de Norteamérica (Litzelman et al., 1998), el MEDUC-RX32 (Huete et al., 2016) y el MEDUC-PG14 (Pizarro et al., 2015), ambos desarrollados en la Pontificia Universidad Católica de Chile. El último instrumento, el MEDUC-PG14, ha sido aplicado en Perú por la Unidad de Educación Médica de la Universidad Peruana Cayetano Heredia, los resultados obtenidos de la aplicación del instrumento MEDUC-PG14 fueron válidos y de alta confiabilidad, demostró ser un instrumento útil y de fácil aplicación para evaluar el desempeño docente en posgrado, en programas clínicos y quirúrgicos, y se recomienda utilizarlo en programas de residencia en países de habla hispana (Flores \& Carbajal, 2021; Olascoaga et al., 2021). Mención aparte merece el instrumento Nation wide Children's Hospital Faculty Clinical Teaching Milestones, el cual es una rúbrica que describe los hitos a alcanzar en 10 áreas de evaluación, donde seis de las áreas se basan en el modelo de Enseñanza Clínica del Desarrollo Docente de Stanford, tres áreas adicionales están dedicadas a preguntas de la encuesta anual para residentes del Accreditation Council for Graduate Medical Education y un área final evalúa el profesionalismo. Este instrumento fue diseñado para evitar los sesgos de las evaluaciones tradicionales de tipo Likert y para proporcionar datos más significativos al docente sobre sus habilidades de enseñanza (Kassis et al., 2017).

\section{Calidad de los instrumentos de evaluación y los sesgos}

La evaluación de la enseñanza clínica y de los profesores debe llevarse a cabo utilizando instrumentos bien diseñados para obtener datos válidos y confiables que puedan utilizarse para respaldar la enseñanza y el aprendizaje, ya sea para proveer un feedback relevante al profesor sobre sus fortalezas y debilidades como para orientar la promoción docente y la distinción de las buenas prácticas. Así mismo, será más confiable si se usan varias fuentes: la autoevaluación, la evaluación por pares y la evaluación realizada por médicos residentes.

Los instrumentos de evaluación tienen riesgo de sesgos, entre ellos podemos citar a los siguientes:

- Miedo a las represalias: Ocurre cuando el residente evaluador continúa a cargo del docente evaluado y teme que si califica de manera justa al profesor le pueda generar algún tipo de antipatía y afectar su formación. En otros casos, debido a que existe una relación de afecto y admiración entre evaluadory evaluado, se genera una pérdida de objetividad.

- Efecto halo: es la tendencia de un evaluador a evaluar ya sea de manera positiva o negativa a alguien basándose solamente en una característica, por ejemplo, calificar bien a un docente en todas las áreas solamente porque es una persona muy amable. El evaluador también puede demostrar lenidad —-blandura o falta de rigor - al calificar al docente; esto sucede cuando un residente o un colega califican bien al docente para no hacerlo sentir mal o para no perjudicar su relación de amistad.

- Falta de motivación: Los evaluadores también pueden experimentar una falta de motivación para realizar las evaluaciones; si estas son voluntarias la tasa de respuesta usual va de 25 a 40\%, lo cual es insuficiente para generar un sistema de evaluación; la motivación también se puede perder cuando el residente no ve un cambio en la conducta del docente a pesar de sucesivas calificaciones. Las evaluaciones que son muy largas y frecuentes pueden conducir a una fatiga en el evaluador, que afectará el cumplimiento y la precisión (Zhou \& Baker, 2014). La mayoría de sesgos ocurre en los residentes de los primeros años (Risucci et al., 1992). Así mismo, se ha visto que los pares realizan evaluaciones más confiables que los residentes (Thomas et al., 2004).

La evaluación debe ser un proceso consciente, donde el evaluador reconoce los aspectos a evaluar y los observa en el evaluado. Cuando esto no ocurre el evaluador puede sesgar la calificación hacia una "nota promedio", que no genera problemas.

\section{Barreras de la evaluación docente}

La cultura de la medicina, clásicamente jerárquica, propone retos para establecer sistemas de evaluación y retroalimentación alumno - profesor. Si bien algunos docentes de una facultad desean ser evaluados y contar con una retroalimentación sobre su labor con fines de mejorar, muchos otros se oponen. En un estudio cualitativo sobre el concepto que tienen los profesores de médicos residentes de lo que es ser "buen profesor", van Roermund y colaboradores señalan, 
“Los profesores realmente creen que podrían hacerlo mejor e indican que quisieran mejorar sus competencias, pero al mismo tiempo, se aferran a las creencias y a los métodos que han aprendido a través de la experiencia" (van Roermund et al., 2011). En este mismo estudio se señala que parte de las dificultades para aceptar las evaluaciones y asumir los cambios responden también a la falta de identidad docente en el profesional médico, problema que tendría sus raíces en la formación médica desde el pregrado, que se perpetúa en un sistema de salud donde el tutor médico se sostiene esencialmente sobre su propio sentimiento vocacional, sin contar con el reconocimiento de su labor tutorial desde todos los puntos de vista: profesional, reserva horaria, estímulo, oportunidades de capacitación docente.

Estos problemas nos plantean la necesidad de hallar soluciones orientadas a crear un sistema de evaluación docente positivo y eficiente, en el que estrategias como la capacitación, el empoderamiento, la obligatoriedad en la participación y la conexión con un plan de desarrollo docente sean elementos claves.

\section{Sistemas de evaluación docente planteados como una cultura organizacional}

A continuación, presentamos estudios sobre experiencias de sistemas de evaluación docente en la residencia médica, donde la retroalimentación estuvo presente.

En el año 2013, Cornelia Fluit y colaboradores publicaron el estudio titulado "Residents provide feedback to their clinical teachers: Reflection through dialogue" (Fluit et al., 2013) donde presenta la experiencia del uso de un instrumento de evaluación de la performance del docente por los residentes y por sí mismo (autoevaluación). El proceso tuvo cuatro pasos: (1) evaluar el desempeño, (2) proporcionar retroalimentación, (3) reflexión y toma de decisiones y (4) usar la retroalimentación para aprender y cambiar. La retroalimentación fue realizada en reuniones entre dos médicos residentes (representantes de los residentes), el profesor evaluado y un profesor moderador, en esta sesión los residentes brindaron el feedback al docente en un ambiente seguro y controlado. El tercer paso fue la reflexión por parte del docente y el planteamiento de una acción. El cuarto paso fue aplicar estos cambios en la práctica real.

Sargeant y colaboradores, por su parte, realizaron un estudio cualitativo sobre la retroalimentación a los docentes, en el que el docente contrastaba su autoevaluación con la evaluación realizada por sus alumnos, encontrando que la retroalimentación estimulaba los procesos de reflexión de los médicos (Sargeant et al., 2009). El proceso de reflexión fue fundamental para la aceptación y el uso de la retroalimentación, lo que sugiere que la reflexión es un enfoque educativo importante en la evaluación formativa y el proceso de retroalimentación y se debe fortalecer como parte de la cultura de una institución educativa.
Keith Baker publicó una experiencia de seis años de evaluación docente y retroalimentación basadas en el residente, en la que los residentes brindaban una retroalimentación escrita a sus docentes cada 6 meses, además de las encuestas numéricas de desempeño docente. Se evidenció que progresivamente los puntajes de calificación de los docentes fueron aumentando en el tiempo y de manera sostenida (Baker, 2010).

\section{Características de los sistemas de mejora en la calidad de la enseñanza y programas de especialización médica}

Con el fin de mejorar la calidad de la enseñanza y de los programas formativos en la residencia médica es necesario contar con un sistema de evaluación docente que cumpla con las siguientes características:

- Es generado por la institución educativa. El sistema de evaluación y desarrollo docente es generado a partir de la misma universidad responsable de la formación del médico residente, donde los docentes deben conocer previamente los roles asignados por la facultad y tener pautas para cumplirlos.

- Las evaluaciones forman parte de la cultura institucional, son periódicas y sostenidas en el tiempo.

- Uso de instrumentos válidos, confiables, factibles de aplicar, que sean conocidos y cuenten con la aceptación de los actores del proceso; combinando la autoevaluación, la evaluación por pares y la evaluación por los alumnos.

- Aplicación del feedback al docente evaluado. Los resultados de las evaluaciones son devueltos al docente mediante un proceso de retroalimentación efectiva, oportuna, específica y significativa al docente evaluado. Esto implica la preparación, la autoevaluación, la retroalimentación y el plan de mejora (Bienstock et al., 2007). La preparación apunta a que el evaluador y el docente evaluado conozcan claramente los objetivos de la evaluación y de la retroalimentación y que ambos reciban capacitación en dar y recibir feedback, para hacerlo de manera positiva, con respeto, tolerancia y humildad. La autoevaluación se refiere a que el docente evaluado debe contar con un instrumento para evaluar su propio desempeño y reflexionar sobre sus logros y dificultades. El feedback puede ser brindado por un jefe directo, por otro docente designado o por un residente.

- Plan de acción de desarrollo docente posterior a la evaluación, enmarcado dentro de la carrera profesional, para ser llevado a cabo en un plazo determinado. 
Conectar los resultados de la evaluación docente con los planes de capacitación es una necesidad de todos los programas de residencia, y es importante mirarlos como una inversión, para que la enseñanza de la medicina asuma, con idoneidad, su responsabilidad frente a la sociedad. La educación médica debe garantizar una atención de la salud oportuna y de calidad. Tener mejores docentes tiene como consecuencia mejores resultados de aprendizaje y mejores resultados en la atención en salud. El desempeño de los docentes de medicina tiene consecuencias en la formación de los residentes, en la atención de los pacientes, en la institución de salud en la que realizan la práctica clínica y en la propia satisfacción personal.

\section{Conclusiones}

El docente en los programas de residentado médico cumple diversos roles como docente y médico asistencial en la institución donde realiza su práctica clínica. Además, influye de manera directa e indirecta en las habilidades que los médicos residentes deben lograr durante su programa de entrenamiento.

Debe ser un objetivo de cada institución universitaria el crear un espacio para el desarrollo del docente, con oportunidades de formación y promoción claramente definidas en base a los logros del docente a lo largo de su vida profesional.

La evaluación de los docentes es fundamental en la profesionalización de la educación médica, debe ser parte de una política institucional, con la participación de diversos actores como son los alumnos, autoridades y los mismos docentes. Se deben aplicar diversos métodos de evaluación y utilizar buenos instrumentos.

El docente evaluado debe conocer el resultado de la evaluación, así como recibir una retroalimentación efectiva. La evaluación debe estar ligada a un plan de capacitación y acción. Esto generará mejores resultados en la formación de los médicos residentes, satisfacción en los docentes y un mayor desarrollo institucional.

Tan importante como desarrollar los planes de mejora, es reconocer y divulgar los resultados óptimos, las prácticas innovadoras y los modelos a seguir.

\section{Referencias}

Arah OA, Hoekstra JBL, Bos AP. \&Lombarts K. (2011) New Tools for Systematic Evaluation of Teaching Qualities of Medical Faculty: Results of an Ongoing Multi-Center Survey. PLoS ONE 6, e25983.

Baker K. (2010). Clinical Teaching Improves with Resident Evaluation and Feedback. Anesthesiology 113, 693-703.
Beckman T, Lee M. \&Mandrekar J. (2004) A comparison of clinical teaching evaluations by resident and peer physicians, Medical Teacher 26, 321-325.

Bienstock JL, Katz NT, Cox SM, Hueppchen N, Erickson S, Puscheck $\mathrm{EE}$, Association of Professors of Gynecology \& Obstetrics Undergraduate Medical Education Committee. (2007). To the point: medical education reviews-providing feedback. American Journal of Obstetrics and Gynecology, 196:508-13.

Finn K, Chiappa V, Puig A. \& Hunt D. (2011) How to become a better clinical teacher: A collaborative peer observation process. Medical Teacher 33, 151-155.

Flores F, Martínez A, Sánchez M, García B. \&Reidl L. (2011). Modelo de competencia docente del profesor de medicina en la UNAM. Revista Electrónica de Investigación y Evaluación Educativa 17, 1- 21.

Flores M. \& Carbajal L. (2021). Evaluación docente en los programas quirúrgicos de residentado médico mediante el instrumento MEDUCPG14. RevistaMédicaHerediana 32,72-78.

Fluit CV, Bolhuis S, Klaassen T, DE Visser M, Grol R, Laan R. \& Wensing M. (2013). Residents provide feedback to their clinical teachers: reflection through dialogue. Medical Teacher 35, e1485-e1492.

Fluit C, Bolhuis S, Grol R, Laan R. \&Wensing M. (2010) Assessing the Quality of Clinical Teachers: A Systematic Review of Content and Quality of Questionnaires for Assessing Clinical Teachers. Journal of General Internal Medicine 25, 1337-45.

Harden RM. \& Crosby J. (2000). AMEE Guide No 20: The good teacher is more than a lecturer the twelve roles of the teacher. Medical Teacher 22, 334-347.

Huete Á, Julio R, Rojas V, Herrera C, Padilla O, Solís N, Pizarro M, Etcheberry L, Sarfatis A, Pérez G, Díaz LA, Delfino A, Muñoz E, Rivera H, Parra DA. \& Bitran M, Riquelme A. (2016). Evaluation of Radiology Teachers' Performance and Identification of the "Best Teachers" in a Residency Program: Mixed Methodology and Pilot Study of the MEDUC-RX32 Questionnaire. Academic Radiology 23, 779-88.

Kassis K, Wallihan R, Hurtubise L, Goode S, Chase M. \& Mahan JD. (2017). Milestone-based tool for learner evaluation of faculty clinical teaching. MedEdPORTAL 13, 10626.

Litzelman D, Stratos GA, Marriott DJ. \&Skeff KM. (1998). Factorial Validation of a Widely Disseminated Educational Framework for Evaluating Clinical Teachers. Academic Medicine 73, 688-95. 
Olascoaga et al.

Matus O, Torres G. \& Parra P. (2009). Utilización del portafolio en Educación Médica. Revista Educación en Ciencias de la Salud 6, 10-19. Newman L, Roberts D. \&Schwartzstein. (2012) Peer Observation of Teaching Handbook. MedEdPORTAL. Accedido en: www.mededportal.org/publication/9150 el 15 de octubre 2021.

Olascoaga AC et al. (2021). Validez y confiabilidad de los MEDUC-PG14 para medir el desempeño docente en la residencia médica en Perú. Revista Médica de Chile 149: 920-927.

Organización Panamericana de la Salud. (2011). Residencias médicas en América Latina. Área de Sistemas de Salud basados en la Atención Primaria de Salud. OPS. Washington, D.C.

Paukert JL. \& Richards BF. (2000). How medical students and residents describe the roles and characteristics of their influential clinical teachers. Academic Medicine 75, 843-845.

Pizarro M, Solís N, Rojas V, Díaz L, Padilla O, Riquelme A, et al. (2015). Evaluación de docentes clínicos de Postgrado: desarrollo y propiedades psicométricas del instrumento. MEDUC-PG14. Revista Médica de Chile, 143, 907-16.

Risucci DA, Lutsky L, Rosati RJ. \&Tortolani AJ. (1992). Reliability and accuracy of resident evaluations of surgical faculty. Evaluation of Health Professions 15, 313-324.

Salehi PP, Jacobs D, Suhail-Sindhu T, Judson BL, Azizzadeh B. \& Lee $\mathrm{YH}$. (2000). Consequences of medical hierarchy on medical students, residents and medical education in otolaryngology. Otolayngology Head and Nech Surgery 163, 906-914.
Sargeant J, Mann KV, Vleuten CP. \&Metsemakers J. (2009). Reflection: a link between receiving and using assessment feedback. Advances in Health Sciences Education 14, 399-410.

Simpson D, Hafler J, Brown D. \& Wilkerson L. (2004). Documentation Systems for Educators Seeking Academic Promotion in U.S. Medical Schools. Academic Medicine 79:783-790.

Stalmeijer RE, Dolmans DH, Wolfhagen IH, Peters WG, van Coppenolle L. \& Scherpbier AJ. (2010). Combined student ratings and self-assessment provide useful feedback for clinical teachers. Advances in Health Sciences Education 15, 315-328.

Vaižgèlienė E, Padaiga Z, Rastenytė D, Tamelis A, Petrikonis K. \&Fluit C. (2017). Evaluation of clinical teaching quality in competency-based residency training in Lithuania. Medicina 53, 339-347.

Van Roermund T, Tromp F, Scherpbier A, Bottema B. \&Bueving $\mathrm{H}$. (2011) Teachers' ideas versus experts' descriptions of 'the good teacher' in postgraduate medical education: implications for implementation. A qualitative study. BMCMedical Education 11, 42-50.

Zhou AY. \& Baker P. (2014). Confounding factors in using upward feedback to assess the quality of medical training: a systematic review. Journal of EducationalEvaluationforHealthProfessions 11, 17. 